\title{
Singular values of the Dirac operator at nonzero density*
}

\section{Takuya Kanazawa}

Department of Physics, University of Regensburg, 93040 Regensburg, Germany ${ }^{\dagger}$

\section{Tilo Wettig}

Department of Physics, University of Regensburg, 93040 Regensburg, Germany

E-mail: tilo.wettig@ur.de

\section{Naoki Yamamoto}

Yukawa Institute for Theoretical Physics, Kyoto University, Kyoto 606-8502, Japan

Institute for Nuclear Theory, University of Washington, Seattle, Washington 98195-1550, USA

Department of Physics, University of Maryland, College Park, MD 20742-4111, USA

E-mail: nyama@umd.edu

\begin{abstract}
At nonzero density the eigenvalues of the Dirac operator move into the complex plane, while its singular values remain real and nonnegative. In QCD-like theories, the singular-value spectrum carries information on the diquark (or pionic) condensate. We have constructed low-energy effective theories in different density regimes and derived a number of exact results for the Dirac singular values, including Banks-Casher-type relations for the diquark (or pionic) condensate, Smilga-Stern-type relations for the slope of the singular-value density, and Leutwyler-Smilgatype sum rules for the inverse singular values. We also present a rigorous index theorem for non-Hermitian Dirac operators.
\end{abstract}

The 30th International Symposium on Lattice Field Theory

June 24 - 29, 2012

Cairns, Australia

\footnotetext{
*Supported by the Alexander von Humboldt Foundation, DFG, and JSPS.

${ }^{\dagger}$ Affiliation until March 2012.

¥ Speaker.
} 


\section{Introduction}

We give a summary of [1], which is very long and sometimes quite technical. Here we present the main ideas and most important results. We work in Euclidean space and in the chiral limit.

\section{Dirac eigenvalues and singular values}

In the presence of a chemical potential $\mu$, the massless Dirac operator in QCD and QCD-like theories is given by

$$
D(\mu)=\gamma_{v} D_{v}+\mu \gamma_{4}=\left(\begin{array}{cc}
0 & D_{L} \\
D_{R} & 0
\end{array}\right)
$$

with $D_{v}=\partial_{v}+i A_{v}$, where $A_{v}=A_{v}^{a} \tau_{a} / 2$ is the gauge field and the $\tau_{a}$ are the generators of the gauge group in the representation in which the fermions transform. The eigenvalue equation and the equation for the singular values are

$$
D(\mu) \psi_{n}=\lambda_{n} \psi_{n} \quad \text { and } \quad D^{\dagger} D \phi_{n}=\xi_{n}^{2} \phi_{n},
$$

where the argument $\mu$ is understood if it is not shown explicitly. For $\mu=0$ we have the trivial relation $\xi_{n}=\left|\lambda_{n}\right|$. For nonzero (and real) $\mu$ the eigenvalues move into the complex plane, while the singular values are always real and nonnegative. Eigenvalues and singular values are unrelated for $\mu \neq 0$ and live on different physical scales. The scale for the eigenvalues is set by the chiral condensate at low density [2] and by the BCS gap $\Delta$ at high density [3], while the scale for the singular values in QCD-like theories is set by the diquark condensate at all densities, see Sec. 3 .

The operators $D(\mu)$ and $D^{\dagger} D$ have the same zero modes, and the same is true for the operators $D(-\mu)$ and $D D^{\dagger}$. For a non-Hermitian Dirac operator the index theorem takes the form

$$
\frac{1}{32 \pi^{2}} \int d^{4} x F \tilde{F}=\frac{1}{2}\left[\operatorname{ind} D(\mu)+\operatorname{ind} D(\mu)^{\dagger}\right]
$$

where ind $D(\mu)=\operatorname{dim} \operatorname{ker} D_{R}-\operatorname{dim} \operatorname{ker} D_{L}$. We generically have ind $D(\mu)=\operatorname{ind} D(\mu)^{\dagger}$ unless the gauge field is fine-tuned. The proof of (2.3) and further properties of the eigenvalues and singular values are given in [1].

\section{Banks-Casher-type relations for the singular values}

For the derivation of the results in this section it is important that the fermionic measure (including source terms) in the partition function is positive definite, see the discussion in [1, App. C]. We restrict ourselves to such cases, which in particular implies an even number $N_{f}$ of flavors (except for adjoint QCD, where $N_{f}$ could also be odd).

\subsection{Two-color QCD}

In two-color QCD the Dirac operator satisfies an anti-unitary symmetry, $\left[C \tau_{2} K, i D\right]=0$ with $C=i \gamma_{4} \gamma_{2}$ the charge conjugation matrix and $K$ the operator of complex conjugation. Adding a 
source term $\frac{1}{2} j \psi^{T} C \gamma_{5} \tau_{2} I \psi+$ c.c. with

$$
I=\left(\begin{array}{cc}
0 & -\mathbb{1}_{N_{f} / 2} \\
\mathbb{1}_{N_{f} / 2} & 0
\end{array}\right)
$$

to the fermionic Lagrangian and using the Nambu-Gor'kov formalism one can show that the partition function takes the form

$$
Z(j)=\left\langle\operatorname{det}^{N_{f} / 2}\left(D^{\dagger} D+j^{2}\right)\right\rangle_{\mathrm{YM}}=\left\langle\prod_{n}\left(\xi_{n}^{2}+j^{2}\right)^{N_{f} / 2}\right\rangle_{\mathrm{YM}},
$$

where YM stands for $N_{f}=0$ in the average over gauge fields. Taking the logarithmic derivative w.r.t. $j$, followed by the limits four-volume $V_{4} \rightarrow \infty$ and $j \rightarrow 0^{+}$(in this order), then yields a relation between the scalar diquark condensate and the density $\rho_{\mathrm{sv}}(\xi)$ of the singular values at the origin,

$$
\left\langle\psi^{T} C \gamma_{5} \tau_{2} I \psi\right\rangle=\frac{N_{f}}{2} \pi \rho_{\mathrm{sv}}(0),
$$

see also [4]. Some subtleties of this result and its derivation are discussed in [1].

\subsection{QCD with isospin chemical potential}

We now consider QCD with $N_{c} \geq 2$ colors, two flavors, and nonzero isospin chemical potential $\mu_{I}=2 \mu$, i.e., we have $D(\mu)$ for the up quark and $D(-\mu)$ for the down quark. Adding a source term $j\left(d_{L}^{\dagger} u_{R}-u_{L}^{\dagger} d_{R}\right)+$ c.c. to the Lagrangian and using $D(\mu)^{\dagger}=-D(-\mu)$ we find

$$
Z(j)=\left\langle\operatorname{det}\left(D^{\dagger} D+j^{2}\right)\right\rangle_{\mathrm{YM}},
$$

which agrees with (3.2) for $N_{f}=2$. After differentiating $\ln Z(j)$ w.r.t. $j$ we obtain a relation between the pionic condensate and $\rho_{\text {sv }}$, i.e.,

$$
\left\langle\bar{u} \gamma_{5} d-\bar{d} \gamma_{5} u\right\rangle=\pi \rho_{\mathrm{sv}}(0)
$$

\subsection{Adjoint QCD}

Turning to QCD with adjoint fermions and any number of colors, we add a source term $\frac{1}{2} j \psi^{T} C \gamma_{5} \psi+$ c.c. and again obtain (3.2). An analogous calculation then yields

$$
\left\langle\psi^{T} C \gamma_{5} \psi\right\rangle=\frac{N_{f}}{2} \pi \rho_{\mathrm{sv}}(0)
$$

\section{Low-energy effective theories with diquark sources}

From now on we focus on two-color QCD and distinguish three density regimes that differ in their pattern of chiral symmetry breaking and in the number of Nambu-Goldstone (NG) modes. 


\subsection{Low density}

This regime was analyzed in great detail in [5, 6]. Here one starts from the symmetry breaking pattern $\mathrm{SU}\left(2 N_{f}\right) \rightarrow \mathrm{Sp}\left(2 N_{f}\right)$ at zero density and treats $\mu$ and the diquark source as a small perturbation. There are $N_{f}\left(2 N_{f}-1\right)-1 \mathrm{NG}$ modes, parametrized by a field $\Sigma=U \Sigma_{d} U^{T}$ with $\Sigma_{d}=\operatorname{diag}(I,-I)$ and $U=\exp \left(i \pi^{a} T^{a} / 2 F\right)$, where the $T^{a}$ are the generators of the coset space $\mathrm{SU}\left(2 N_{f}\right) / \mathrm{Sp}\left(2 N_{f}\right)$ and $F$ is a low-energy constant (LEC). The leading-order effective Lagrangian in the chiral limit is

$$
\mathscr{L}_{\text {eff }}^{\mathbf{L}}=\frac{F^{2}}{2} \operatorname{tr}\left(\nabla_{v} \Sigma \nabla_{v} \Sigma^{\dagger}\right)-\Phi_{\mathbf{L}} \operatorname{Re} \operatorname{tr}(\overline{J \Sigma})
$$

with

$$
\begin{array}{rlrl}
\nabla_{v} \Sigma & =\partial_{v} \Sigma-\mu \delta_{v 0}(B \Sigma+\Sigma B), & \nabla_{v} \Sigma^{\dagger}=\partial_{v} \Sigma^{\dagger}+\mu \delta_{v 0}\left(\Sigma^{\dagger} B+B \Sigma^{\dagger}\right), \\
B & =\left(\begin{array}{cc}
\mathbb{1}_{N_{f}} & 0 \\
0 & -\mathbb{1}_{N_{f}}
\end{array}\right), & \bar{J} & =\left(\begin{array}{cc}
J_{L} & 0 \\
0 & -J_{R}^{\dagger}
\end{array}\right) .
\end{array}
$$

Here, $J_{L / R}$ are antisymmetric complex matrices of dimension $N_{f}$ in flavor space, and $\Phi_{\mathbf{L}}$ is another LEC equal to the diquark condensate per flavor and handedness at $\mu=0$ and without sources,

$$
\Phi_{\mathbf{L}}=\frac{1}{N_{f}}\left|\left\langle\psi_{i}^{T} C \tau_{2} I \psi_{i}\right\rangle\right|_{\bar{J}=0, \mu=0} \quad(i=L, R) .
$$

For $J_{R}=-J_{L}=j I$ there are two types of NG modes [6],

$$
\begin{array}{lll}
\text { type 1: } & \text { mass }=\sqrt{j \Phi_{\mathbf{L}} / F^{2}} & \left(N_{f}^{2}-N_{f}-1 \text { modes }\right), \\
\text { type 2: } & \text { mass }=\sqrt{j \Phi_{\mathbf{L}} / F^{2}+(2 \mu)^{2}} & \left(N_{f}^{2} \text { modes }\right) .
\end{array}
$$

The type- 2 modes become massive for $\mu \neq 0$, while the type- 1 modes stay massless for $j \rightarrow 0$.

\subsection{Intermediate density}

At intermediate density $\mu$ can no longer be treated as a small perturbation and breaks the original $\mathrm{SU}\left(2 N_{f}\right)$ symmetry to $\mathrm{SU}\left(N_{f}\right)_{L} \times \mathrm{SU}\left(N_{f}\right)_{R} \times \mathrm{U}(1)_{B}$. A diquark condensate then breaks this symmetry to $\mathrm{Sp}\left(N_{f}\right)_{L} \times \mathrm{Sp}\left(N_{f}\right)_{R}$. The corresponding NG modes are $\Sigma_{L}, \Sigma_{R} \in \mathrm{SU}\left(N_{f}\right) / \operatorname{Sp}\left(N_{f}\right)$ and $V \in \mathrm{U}(1)_{B}$, and the total number of NG modes in this regime is $N_{f}\left(N_{f}-1\right)-1$. The $\mathrm{U}(1)_{A}$ symmetry is broken explicitly by the anomaly. The effective Lagrangian is

$$
\mathscr{L}_{\text {eff }}^{\mathbf{I}}=\left\{\begin{array}{cc}
N_{f} f_{0}^{2}\left[\left|\partial_{0} V\right|^{2}+v_{0}^{2}\left|\partial_{i} V\right|^{2}\right]+\frac{f^{2}}{2} \operatorname{tr}\left[\left|\partial_{0} \Sigma_{L}\right|^{2}+v^{2}\left|\partial_{i} \Sigma_{L}\right|^{2}+(L \leftrightarrow R)\right] \\
-\Phi_{\mathbf{I}} \operatorname{Re}\left[V \operatorname{tr}\left(J_{L} \Sigma_{L}-J_{R} \Sigma_{R}\right)\right] & \text { for } N_{f} \geq 4, \\
2 f_{0}^{2}\left[\left|\partial_{0} V\right|^{2}+v_{0}^{2}\left|\partial_{i} V\right|^{2}\right]+2 \Phi_{\mathbf{I}} \operatorname{Re}\left[\left(j_{L}-j_{R}\right) V\right] & \text { for } N_{f}=2,
\end{array}\right.
$$

where $f_{0}, f$ and $v_{0}, v$ are LECs corresponding to the decay constants and velocities of the NG modes, respectively, and $\Phi_{\mathrm{I}}$ is an LEC similar to (4.4) but with $\mu \neq 0$. All LECs depend on $\mu$. The masses of the NG modes are given by

$$
m_{A}=\sqrt{j \Phi_{\mathbf{I}} / f_{A}^{2}} \text { for } A=0, \ldots, N_{f}\left(N_{f}-1\right)-2,
$$

where $f_{A}=f$ for $A \geq 1$. Note that in this regime all NG modes are massless in the $j \rightarrow 0$ limit. 


\subsection{High density}

At very high density the $\mathrm{U}(1)_{A}$ anomaly is suppressed due to the screening of instantons [7, 8]. The $\mathrm{U}(1)_{A}$ symmetry of the action is no longer broken explicitly by the anomaly but spontaneously by the diquark condensate. Therefore the symmetry-breaking pattern is now [9]

$$
\mathrm{SU}\left(N_{f}\right)_{L} \times \mathrm{SU}\left(N_{f}\right)_{R} \times \mathrm{U}(1)_{B} \times \mathrm{U}(1)_{A} \rightarrow \mathrm{Sp}\left(N_{f}\right)_{L} \times \mathrm{Sp}\left(N_{f}\right)_{R} .
$$

The NG modes are the same as at intermediate density, except that there is an additional NG mode $A \in \mathrm{U}(1)_{A}$ which can be considered to be the $\eta^{\prime}$ whose mass has become small. Hence the total number of NG modes in this regime is $N_{f}\left(N_{f}-1\right)$. The effective Lagrangian is

$$
\mathscr{L}_{\mathrm{eff}}^{\mathbf{H}}= \begin{cases}{\left[\frac{N_{f} \tilde{f}_{0}^{2}}{2}\left(\left|\partial_{0} L\right|^{2}+\tilde{v}_{0}^{2}\left|\partial_{i} L\right|^{2}\right)+\frac{\tilde{f}^{2}}{2} \operatorname{tr}\left(\left|\partial_{0} \Sigma_{L}\right|^{2}+\tilde{v}^{2}\left|\partial_{i} \Sigma_{L}\right|^{2}\right)+(L \leftrightarrow R)\right]} & \\ -\Phi_{\mathbf{H}} \operatorname{Re} \operatorname{tr}\left(J_{L} L \Sigma_{L}-J_{R} R \Sigma_{R}\right)-\frac{2 \tilde{f}_{0}^{2}}{N_{f}} m_{\text {inst }}^{2} \operatorname{Re}\left(L^{\dagger} R\right)^{N_{f} / 2} & \text { for } N_{f} \geq 4, \\ \tilde{f}_{0}^{2}\left[\left|\partial_{0} L\right|^{2}+\tilde{v}_{0}^{2}\left|\partial_{i} L\right|^{2}+(L \leftrightarrow R)\right]+2 \Phi_{\mathbf{H}} \operatorname{Re}\left(j_{L} L-j_{R} R\right)-\tilde{f}_{0}^{2} m_{\text {inst }}^{2} \operatorname{Re}\left(L^{\dagger} R\right) & \text { for } N_{f}=2,\end{cases}
$$

where $L=A^{\dagger} V$ and $R=A V$. Similar to Sec. 4.2 we have LECs $\tilde{f}_{0}, \tilde{f}$ and $\tilde{v}_{0}, \tilde{v}$ as well as $\Phi_{\mathbf{H}}$, all of which depend on $\mu$. The term involving $m_{\text {inst }}$ in (4.9) corresponds to the single-instanton contribution to the $\eta^{\prime}$ mass, with $m_{\text {inst }} \rightarrow 0$ as $\mu \rightarrow \infty$, see [1] for a detailed discussion. The masses of the NG modes are given by

$$
\begin{aligned}
& \text { type 1: } \quad m_{A}=\sqrt{j \Phi_{\mathbf{H}} / \tilde{f}_{A}^{2}} \quad\left(N_{f}^{2}-N_{f}-1 \text { modes }\right) \text {, } \\
& \text { type 2: } \quad m_{\eta^{\prime}}=\sqrt{j \Phi_{\mathbf{H}} / \tilde{f}_{0}^{2}+m_{\text {inst }}^{2}} \quad \text { (1 mode). }
\end{aligned}
$$

The type- 1 modes stay massless for $j \rightarrow 0$, while the $\eta^{\prime}$ becomes massive as $\mu$ is lowered.

The effective theory at intermediate density can be obtained from the effective theory at low density (or high density) by integrating out the NG modes that become massive as the density is increased (or decreased). In this way the LECs of the three different regimes can be matched, see [1] for details. The domains of validity of the three effective theories, and their overlaps, are also discussed in [1].

\section{Smilga-Stern-type relations for the singular values}

Following the approach of Smilga and Stern for QCD at zero density [10], we can compute the slope of the singular-value density at the origin. For technical reasons we start at infinite density.

\subsection{Infinite density}

We now add a more general source term $\frac{1}{2} \psi^{T} C \gamma_{5} \tau_{2} J \psi+$ c.c. to the fermionic Lagrangian, where $J=J_{R}=-J_{L}$ is again an antisymmetric $N_{f} \times N_{f}$ matrix, which we can decompose as

$$
J=I \sum_{A=0}^{N} j_{A} t^{A}=j I+I \sum_{a=1}^{N} j_{a} t^{a} \quad \text { with } \quad N=\frac{1}{2} N_{f}\left(N_{f}-1\right)-1 .
$$


Here, the $t^{A}$ are the generators of $\mathrm{U}\left(N_{f}\right) / \mathrm{Sp}\left(N_{f}\right)$ and the $j_{A}$ are real parameters with $j_{0}=j \sqrt{N_{f}}$. We can then show the partition function of two-color QCD is given by

$$
Z(J)=\left\langle\operatorname{det}^{1 / 2}\left(D^{\dagger} D+J^{\dagger} J\right)\right\rangle_{\mathrm{YM}}=\left\langle\prod_{n} \operatorname{det}^{1 / 2}\left(\xi_{n}^{2}+J^{\dagger} J\right)\right\rangle_{\mathrm{YM}} .
$$

For $N_{f} \geq 4$ we define the scalar susceptibility

$$
K_{a b}(j)=\left.\lim _{V_{4} \rightarrow \infty} \frac{1}{V_{4}} \partial_{j_{a}} \partial_{j_{b}} \ln Z(J)\right|_{\text {all } j_{a}=0},
$$

for which we obtain after some algebra

$$
K_{a b}(j)=\delta_{a b} \lim _{V_{4} \rightarrow \infty} \frac{1}{V_{4}}\left\langle\sum_{n} \frac{\xi_{n}^{2}-j^{2}}{\left(\xi_{n}^{2}+j^{2}\right)^{2}}\right\rangle_{j}=\delta_{a b} \int_{0}^{\infty} d \xi \rho_{\mathrm{sv}}(\xi ; j) \frac{\xi^{2}-j^{2}}{\left(\xi^{2}+j^{2}\right)^{2}} \sim \delta_{a b} \rho_{\mathrm{sv}}^{\prime}(0) \ln \frac{\tilde{\Lambda}}{j}
$$

In the last step we cut off the integral at $\xi=\tilde{\Lambda}$ and extracted the part that diverges for $j \rightarrow 0$. On the low-energy effective theory side we start from (4.9), where at infinite density we can set $m_{\text {inst }}=0$. Performing a one-loop calculation with a momentum cutoff $\tilde{\Lambda}$ we obtain for $j \rightarrow 0$

$$
K_{a b}(j) \sim \delta_{a b}\left[\frac{\left(N_{f}-4\right)\left(N_{f}+2\right)}{2 N_{f} \tilde{f}^{4}}+\frac{2}{N_{f} \tilde{f}_{0}^{2} \tilde{f}^{2}}\right] \frac{\Phi_{\mathbf{H}}^{2}}{16 \pi^{2}} \ln \left(\frac{\tilde{\Lambda}}{j}\right) .
$$

Matching the divergences of (5.4) and (5.5) for $j \rightarrow 0$ yields the slope of $\rho_{\mathrm{sv}}$ at the origin,

$$
\rho_{\mathrm{sv}}^{\prime}(0)=\left[\frac{\left(N_{f}-4\right)\left(N_{f}+2\right)}{2 N_{f} \tilde{f}^{4}}+\frac{2}{N_{f} \tilde{f}_{0}^{2} \tilde{f}^{2}}\right] \frac{\Phi_{\mathbf{H}}^{2}}{16 \pi^{2}} .
$$

This method does not work for $N_{f}=2$, but we expect, based on experience from partially quenched chiral perturbation theory [11], that (5.6) is also valid for $N_{f}=2$.

\subsection{Intermediate density}

At intermediate density the calculation on the low-energy effective theory side starts from (4.6) and proceeds in a similar way to yield

$$
\rho_{\mathrm{sv}}^{\prime}(0)=\left[\frac{\left(N_{f}-4\right)\left(N_{f}+2\right)}{2 N_{f} f^{4}}+\frac{1}{N_{f} f_{0}^{2} f^{2}}\right] \frac{\Phi_{\mathbf{I}}^{2}}{16 \pi^{2}} .
$$

\subsection{Zero density}

At strictly zero density we start from (4.1) with $\mu=0$ and obtain in a similar way

$$
\rho_{\mathrm{sv}}^{\prime}(0)=\frac{\left(N_{f}-2\right)\left(N_{f}+1\right)}{N_{f} F^{4}} \frac{\Phi_{\mathbf{L}}^{2}}{16 \pi^{2}}
$$

\subsection{Relation between the three results}

At first sight it does not seem possible to interpolate smoothly between the three results for $\rho_{\mathrm{sv}}^{\prime}(0)$ at zero, intermediate, and infinite density. This puzzle can be understood by analogy with the interpolation between $\mathrm{SU}(2)$ and $\mathrm{SU}(3)$ chiral perturbation theory, where the strange quark mass plays the role of the symmetry-breaking parameter. In our case this parameter is $\mu$. We find (see [1] for details) that at low density the slope is given by (5.7) for $\xi \ll \mu^{2} / \Lambda$ and by (5.8) for $\mu^{2} / \Lambda \ll \xi \ll \Lambda$, where $\Lambda \sim F \sim \Phi_{\mathbf{I}}^{1 / 3}$. At high density the slope is given by (5.7) for $\xi \ll g m_{\eta^{\prime}}^{2} / \Delta$ and by (5.6) for $g m_{\eta^{\prime}}^{2} / \Delta \ll \xi \ll g \Delta$, where $g$ is the coupling constant. 


\section{Finite-volume analysis}

In a finite volume $V_{4}=L^{4}$, the three low-energy effective theories constructed in Sec. 4 have $\varepsilon$-regimes in which the theory becomes zero-dimensional. The corresponding condition is

$$
\frac{1}{m_{\ell}} \ll L \ll \frac{1}{m_{\mathrm{NG}}},
$$

where $m_{\ell}$ is the mass scale of the lightest non-NG particle (see [1, Sec. 5.5] for actual values) and $m_{\mathrm{NG}}$ is the mass scale of the NG particles, see Eqs. (4.5), (4.7), and (4.10). In these $\varepsilon$-regimes we have derive Leutwyler-Smilga-type sum rules [12] for the inverse singular values and random matrix theories from which microscopic singular-value correlation functions can be derived [1].

\section{Summary}

We have derived a number of exact results for the singular-value spectrum in QCD-like theories without a sign problem. Our results could be used in lattice simulations to obtain the diquark condensate at any density, allowing for a numerical test of the conjectured BEC-BCS crossover.

\section{References}

[1] T. Kanazawa, T. Wettig, and N. Yamamoto, Singular values of the Dirac operator in dense QCD-like theories, JHEP 12 (2011) 007 [arXiv: 1110.5858 ].

[2] T. Banks and A. Casher, Chiral Symmetry Breaking in Confining Theories, Nucl. Phys. B169 (1980) 103.

[3] N. Yamamoto and T. Kanazawa, Dense QCD in a Finite Volume, Phys. Rev. Lett. 103 (2009) 032001 [arXiv:0902.4533].

[4] K. Fukushima, Characteristics of the eigenvalue distribution of the Dirac operator in dense two-color QCD, JHEP 07 (2008) 083 [arXiv: 0806.1104 ].

[5] J. B. Kogut, M. A. Stephanov, and D. Toublan, On two-color QCD with baryon chemical potential, Phys. Lett. B464 (1999) 183 [hep-ph/9906346].

[6] J. B. Kogut, M. A. Stephanov, D. Toublan, J. J. M. Verbaarschot, and A. Zhitnitsky, QCD-like theories at finite baryon density, Nucl. Phys. B582 (2000) 477 [hep-ph/ 0001171$].$

[7] T. Schafer, Instanton effects in QCD at high baryon density, Phys. Rev. D65 (2002) 094033 [hep-ph/0201189].

[8] T. Schäfer, QCD and the eta' mass: Instantons or confinement?, Phys. Rev. D67 (2003) 074502 [hep-lat/0211035].

[9] T. Kanazawa, T. Wettig, and N. Yamamoto, Chiral Lagrangian and spectral sum rules for dense two-color QCD, JHEP 08 (2009) 003 [arXiv: 0906.3579 ].

[10] A. V. Smilga and J. Stern, On the spectral density of Euclidean Dirac operator in QCD, Phys. Lett. B318 (1993) 531.

[11] D. Toublan and J. J. M. Verbaarschot, The spectral density of the QCD Dirac operator and patterns of chiral symmetry breaking, Nucl. Phys. B560 (1999) 259 [hep-th/9904199].

[12] H. Leutwyler and A. V. Smilga, Spectrum of Dirac operator and role of winding number in QCD, Phys. Rev. D46 (1992) 5607. 